\title{
TERAPIA OCUPACIONAL PRECOZ E INTENSIVA EN LA PREVENCIÓN DEL DELIRIUM EN ADULTOS MAYORES INGRESADOS A UNIDADES DE PACIENTE CRÍTICO. ENSAYO CLÍNICO RANDOMIZADO: RESULTADOS PRELIMINARES. ${ }^{1}$
}

\author{
EARLY AND INTENSIVE OCCUPATIONAL THERAPY IN PREVENTION OF \\ DELIRIUM IN OLDER ADULTS ADMITTED TO CRITICAL PATIENT UNIT. \\ RANDOMIZED CLINICAL TRIAL: \\ PRELIMARY RESULTS
}

\author{
Evelyn Álvarez E ${ }^{2,3}$, , . Maricel Garrido $\mathrm{M}^{1}$, Francisco González A. ${ }^{5}$, Elisabeth Guzmán ${ }^{1}$, \\ Tatiana Donoso G ${ }^{1}$, Sebastián Gallegos B ${ }^{1}$, Sebastián Vergara R. ${ }^{1}$, Rolando Aranda G. ${ }^{1}$, \\ Stephanie Prieto S. ${ }^{1}$, Constanza Briceño R ${ }^{1}$, Eduardo Tobar A. ${ }^{4}$, Consuelo Alzamora M., Camila \\ Bolvarán E., Camila Concha R., Fernanda Valencia Q., FabiolaVillalobos G.
}

\section{RESUMEN}

Objetivo: Comparar la eficacia de la prevención no farmacológica estándar (PnFE) versus la prevención no farmacológica reforzada (PnFR), consistente en prevención no farmacológica estándar más Terapia Ocupacional (TO) precoz e intensiva, en la incidencia del delirium en adultos mayores (AM) ingresados a unidad de pacientes críticos (UPC).

Diseño: Ensayo clínico randomizado, en UPC del Hospital Clínico de la Universidad de Chile (HCUCH).

Sujetos: 70 pacientes de edad igual o superior a 60 años, ingresados al HCUCH entre abril y octubre del 2011, con necesidad de ingreso a UPC para monitorización, hospitalización por enfermedad aguda/crónica descompensada, con consentimiento del paciente o familiar y sin presencia de delirium al ingreso ni deterioro cognitivo previo al estudio.

Materiales y Métodos: PnFE (grupo control) consiste en: reorientación, movilización precoz, corrección de déficit sensoriales, manejo ambiental, protocolo de sueño y reducción de fármacos anticolinérgicos, versus PnFR (grupo experimental), que considera las siguientes áreas de intervención de TO: estimulación polisensorial, posicionamiento, estimulación cognitiva, entrenamiento en actividades de la vida diaria básica, estimulación motora de extremidades superiores y participación familiar; durante 5 días, dos veces al día. Se evaluó la presencia del delirium, con el CAM dos veces al día durante 5 días, y la severidad de éste con DRS; previo al alta se evaluó, independencia funcional con FIM, estado cognitivo con MMSE y fuerza de garra con dinamómetro de Jamar.

Resultados: La PnFR de TO se asocia a menor incidencia de delirium, afectando al 16,1\% del grupo con prevención no farmacológica estándar versus un 3,1\% del con prevención no farmacológica reforzada, así como a menos días de hospitalización (20,6 días versus 10,4 p=.009). La independencia funcional al alta se mantiene en aspectos cognitivos $(32,5$ versus 32,9$)$ mientras que en aspectos motores aumenta significativamente $(46,5$ versus 58,31 , $\mathrm{P}=.03)$.

Conclusión: La intervención precoz e intensiva de TO es efectiva en la prevención del delirium en AM hospitalizados, reduce su estadía y aumenta niveles de independencia funcional motora al alta.

Palabras claves: Delirium, Adulto Mayor, Terapia Ocupacional, Unidad de Pacientes Críticos, Prevención no farmacológica.

\footnotetext{
${ }^{1}$ Esta publicación es producto de investigación desarrollada en el marco del Proyecto FONIS No SA10I20015

${ }^{2}$ Servicio de Medicina Física y Rehabilitación , Hospital Clínico Universidad de Chile

${ }^{3}$ Escuela de Terapia Ocupacional. Universidad de Chile

${ }^{4}$ Terapeuta Ocupacional, evalvarez@med.uchile.cl

5 Unidad de Paciente Crítico, Hospital Clínico Universidad de Chile
} 


\section{ABSTRACT}

Objective: To compare the efficacy of standard non pharmacological prevention of delirium versus intensified prevention of delirium (standard prevention plus early and intensive Occupational Therapy) in the incidence of delirium in older adults (OA) admitted to critical patient unit (CPU).

Desing: Randomized control trial, blinded to outcome evaluator, in the CPU of Hospital Clínico Universidad de Chile.

Subjects: 70 patients aged 60 years or older, admitted to CPU between April and October of 2011, with need for admission to CPU for monitoring, acute or decompensated chronic illness, without cognitive impairment and consent by patient or family member.

Materials and Methods: Standard prevention group consisted in: reorienting, early mobilization, correction of sensory deficit, environmental management, protocol of sleep and reduction of drugs, and intensified prevention based on standard measured plus early and intensive Occupational Therapy: multisensory stimulation, positioning, cognitive stimulation, training in activities of daily living, motor stimulation of the upper extremities and family participation, twice a day for 5 days. Delirium was evaluated (twice a day for 5 days) with CAM and severity with DRS. Primary outcome was delirium incidence, and secondarily were functional independence (FIM), cognitive status (MMSE) and strength of grip with Jamar dynamometer at leaving.

Results: Early intervention and intensive occupational therapy is associated with lower incidence of delirium, affecting $16.1 \%$ of non-pharmacological standard prevention group and $3.1 \%$ of intensified prevention group, as well as fewer days of hospitalization (20,6 days versus $10,4, \mathrm{p}=0,009)$. The functional independence at leaving keeps in cognitive (32.5 versus 32.9 ) and is increases significantly in motor aspects (46.5 versus $58.31, \mathrm{P}=.03$ ).

Conclusion: Standard prevention plus early intensive intervention of Occupational Therapy is effective in preventing delirium in hospitalized OA, reduces their stay and increases levels of functional independence at leaving.

Keywords: Delirium, Older Adults, Occupational Therapy, Critical Patient Unit, Non-pharmacological prevention. 


\section{INTRODUCCIÓN}

Uno de los objetivos sanitarios del país es enfrentar los desafíos derivados del envejecimiento de la población. Según el Instituto Nacional de Estadística (INE) los AM en Chile representan el $11,5 \%$ de la población total y se espera que al 2020 un 17,3\% de los chilenos sea mayor de 60 años (CENSO 2002).

Los AM tienen una mayor prevalencia de enfermedades crónicas no transmisibles, más episodios de enfermedades agudas y mayor riesgo de accidentes que el resto de la población, presentando en Chile altas tasas de hospitalizaciones, alcanzando una proporción 2.6 veces mayor que la población general y un promedio de 9,2 días de estadía hospitalaria, en comparación con 5 días de estadía de la población general (estudio SENAMA 2009). Otro dato importante, es que las hospitalizaciones de los AM al año 2002, generó un 50\% del gasto en salud en dicha población y que para el año 2020 será 2,5 veces mayor, según el estudio del “impacto del Envejecimiento en el gasto en salud" (Chile, Superintendencia de Salud, 2002-2020).

Una de las complicaciones más frecuentes de los AM hospitalizados es la aparición de delirium, siendo considerado un factor desfavorable en la evolución hospitalaria. El delirium, también llamado síndrome confusional agudo, es un trastorno que ha sido descrito según el DSM IV (1994), como un cuadro clínico de inicio agudo y curso fluctuante, el cual se caracteriza por generar alteraciones de la conciencia, de la atención y del pensamiento. Además, pueden aparecer ideas delirantes y alucinaciones. Constituye un síndrome geriátrico relevante en la población de adultos mayores (AM), tanto por su prevalencia e impacto como por corresponder en muchas ocasiones a la única expresión de enfermedad sistémica, cerebral o intoxicación (Lipowski, 1987).

En cuanto a su etiología, no es posible definir un único factor desencadenante, ya que suele ser el resultado de la concurrencia de factores de riesgo modificables y no modificables. Dentro de los factores no modificables se encuentra: la demencia, historia previa de delirium, deterioro cognitivo, enfermedades neurológicas, múltiples comorbilidades, insuficiencia renal crónica y enfermedad hepática. Dentro de los factores modificables destaca: el déficit sensorial, inmovilización, uso de medicamentos (sedantes, anticolinérgicos, polifarmacia, retirada de alcohol o drogas), enfermedades neurológicas agudas, enfermedades intercurrentes, trastorno metabólico, cirugía, ambiente clínico, dolor, angustia emocional y privación de sueño (Inouye, 1999).

A partir de los factores de riesgo señalados se puede mencionar que el grupo de pacientes con mayor riesgo de presentar delirium sería el constituido por AM que presentan algún grado de deterioro cognitivo previo, con patología médica añadida y que están expuestos a diversos factores de riesgos intrínsecos y extrínsecos del proceso mórbido y de su tratamiento. Por lo tanto, cuantos más factores de riesgo confluyan, más probable será la aparición del delirium (Fong, 2009).

La importancia de estos factores tendría relación con su manejo, ya que se ha planteado que entre el $30 \%$ y $40 \%$ de los casos de delirium son prevenibles y además que la prevención es la estrategia más eficaz para reducir al mínimo la aparición de delirium y sus resultados adversos (González, 2003). 
Considerando las características de esta población, el delirium tiene incidencias estimadas entre un 14-56\% en los AM hospitalizados, aumentando el riesgo en pacientes de unidades postoperatorias (cardiotoráxica, emergencia traumatológica, vasculares, cataratas), como en el caso de pacientes sometidos a cirugía de cadera en los que el delirium tiene una incidencia que varía entre un 16-62\% (Inouye, 2000). Las unidades donde se describe una mayor incidencia de delirium son las de cuidados intensivos, las de pacientes subagudos o las de cuidados paliativos, alcanzando valores entre 70-87 \% (Inouye, 2000; McDowell, 1998).

Su subdiagnóstico es habitual y la intervención tardía o ausencia de manejo puede generar resultados tales como: aumento de la comorbilidad y mortalidad (Pessoa, 2010; González, 2009; Miller, 2008), prolongación de la estancia hospitalaria, compromiso cognitivo y funcional (Guerini, 2010; Rudolph, 2010), produciendo un significativo impacto en la calidad de vida de los AM junto con el aumento en los costos de salud (Eamonn, 2010; Leslie 2008). Además, recientemente se ha planteado que la duración del delirium también impacta en los resultados, González et al (2099), encontraron que la mortalidad aumenta un 11\% por cada 48 horas de delirium.

Teniendo en cuenta estos elementos podemos decir que el delirium en el AM hospitalizado es un problema de salud relevante para dicha población. Así, la elaboración de intervenciones sistematizadas que ayuden a la prevención o disminución de días de delirium durante la hospitalización, tendrá un gran impacto en la salud y calidad de vida tanto de ellos como de sus familiares.

Por otro lado, estudios señalan que la Terapia Ocupacional (TO) a nivel hospitalario ha generado un impacto positivo en áreas afines, como mejorar la independencia funcional en las actividades de la vida diaria (AVD) y disminuir el riesgo de deterioro en la ejecución de estas habilidades en pacientes con ACV (Legg, 2007). También se han demostrado beneficios en el mantenimiento de habilidades involucradas en la realización de actividades de autocuidado en pacientes operados por fractura de cadera que reciben intervención temprana de TO y que a dos meses muestran mejor manejo en la comunidad en comparación con quienes no la reciben (Hagsten, 2004). En pacientes sometidos a ventilación mecánica en UPC, la intervención precoz de T.O más terapia física ha mostrado una mejora en la independencia funcional al alta, disminución de la incidencia del delirium (Schweickert, 2009) y de los días de estadía hospitalaria en dicha población (Needham, 2010). Pese a lo anterior, no existen precedentes de investigaciones dirigidas a prevención y manejo de delirium en UPC. Por lo tanto, es sustentable el desarrollo de proyectos que exploren el impacto de la intervención de TO en la prevención del delirium, la disminución de su duración y de días de hospitalización en los AM.

El objetivo del estudio es comparar la eficacia de la prevención no farmacológica estándar (PnFE) versus la prevención no farmacológica reforzada (PnFR), consistente en prevención no farmacológica estándar más Terapia Ocupacional (TO) precoz e intensiva, en la incidencia del delirium en adultos mayores (AM) ingresados a unidad de pacientes críticos (UPC).

\section{Metodología}


Para la investigación se utilizó una metodología cuantitativa, del tipo ensayo clínico randomizado. Se compararon los resultados de dos estrategias de prevención del delirium: la prevención no farmacológica estándar (PnFE) respecto de la prevención no farmacológica reforzada $(\mathrm{PnFR})$, esta última consiste en prevención no farmacológica estándar más terapia ocupacional precoz e intensiva.

La muestra se obtuvo en base a los pacientes AM ingresados a UPC del Hospital Clínico de la Universidad de Chile, reclutados entre los meses de abril y octubre del año 2011, alcanzando un $\mathrm{n}=70$.

Criterios de inclusión: edad igual o superior a 60 años al ingreso hospitalario, tener $\leq 24$ horas en la UPC, presentar necesidad de ingreso a UPC para monitorización, enfermedad aguda o crónica descompensada y contar con consentimiento por parte del paciente o un familiar relacionado.

Criterios de exclusión: pacientes con deterioro cognitivo previo a la admisión (medido con Test del Informador-TIN), trastorno severo de la comunicación, limitante cultural del lenguaje (idioma distinto al español), delirium al ingreso a UPC o previo al inicio de la intervención, necesidad de ventilación mecánica invasiva, limitación precoz del esfuerzo terapéutico y presencia de comorbilidad mayor con mortalidad esperada dentro de 90 días (en opinión del equipo tratante).

Para resguardar principios éticos y de bioseguridad, de presente estudio, se implementó proceso de obtención del consentimiento informado por parte del propio paciente o de un familiar responsable, a fin de proteger sus intereses y el principio de autonomía. Asimismo, la intervención se adhiere al principio ético de no maleficencia, al proponer técnicas validadas y que no representan riesgos para los participantes. También responde al concepto bioético de justicia distributiva, ya que ambos grupos en estudio recibirán atenciones de salud preventiva que contemplan un alto respaldo en la bibliografía médica. Estas intervenciones, tienden a disminuir la condición de vulnerabilidad, respondiendo a las necesidades y prioridades de salud de los AM que se hospitalizan.

La PnFE consiste en: reorientación (hora, fecha, lugar y motivo de hospitalización), movilización precoz, corrección de déficit sensoriales (uso de ayudas técnicas: lentes, audífonos), manejo ambiental (instalación de un reloj y calendario en la sala del paciente y evitar contenciones físicas), protocolo de sueño (disminuir luces, ruido y administración de fármacos nocturnos), reducción de fármacos con potencial anticolinérgico y minimizar el empleo de benzodiacepinas. Para ello se capacitó al equipo médico, de enfermería y de auxiliares de la UPC en temáticas como: delirium en el AM (causas, tipos), factores de riesgo, síntomas prodrómicos y funciones de cada integrante del equipo en la prevención.

La PnFR consiste en PnFE más la intervención precoz e intensiva de TO, la cual consiste en: estimulación polisensorial, posicionamiento, estimulación cognitiva, entrenamiento en actividades de la vida diaria básicas (AVDB), estimulación motora de extremidades superiores (EMEESS) y participación de los familiares (Tabla $\mathrm{N}^{\circ} 1$ ). Esta se realizó por un período de cinco días con una frecuencia de dos intervenciones diarias, de 40 minutos de duración cada una. 
Tabla 1. Resumen de la prevención no farmacológica reforzada (PnFE más TO precoz e intensiva).

\begin{tabular}{|l|l|}
\hline \multicolumn{1}{|c|}{ INTERVENCIÓN } & \multicolumn{1}{|c|}{ DESCRIPCIÓN } \\
\hline Estimulación Polisensorial & $\begin{array}{l}\text { Estimulación intensa y regulada a los diferentes canales sensoriales del } \\
\text { paciente (visual, auditivo, táctil, propioceptivo y gustativo) con el fin de } \\
\text { incrementar el estado de alerta, prevenir la deprivación sensorial y aumentar } \\
\text { la interacción del paciente con los objetos y personas que lo rodean. }\end{array}$ \\
\hline Posicionamiento & $\begin{array}{l}\text { Implementación precoz de aditamentos como cuñas antiedema, topes } \\
\text { antiequino y adaptaciones que dejen libre de presión los sitos de mayor } \\
\text { frecuencia de escaras, con el fin de prevenir la rigidez articular, aparición de } \\
\text { ulceras por presión y entregar confort al paciente. }\end{array}$ \\
\hline Estimulación cognitiva & $\begin{array}{l}\text { Intervención utilizada para mantener activas las funciones mentales, tales } \\
\text { como: conciencia, orientación, atención, memoria, cálculo, praxis y lenguaje, } \\
\text { a través de protocolos de estimulación cognitiva y diálogo con el paciente. }\end{array}$ \\
\hline $\begin{array}{l}\text { Entrenamiento en } \\
\text { actividades de la vida } \\
\text { diaria básica (AVDB) }\end{array}$ & $\begin{array}{l}\text { Intervención que busca promover la realización independiente de las AVDB } \\
\text { de higiene, arreglo personal y alimentación. En aquellos pacientes con mayor } \\
\text { nivel de independencia se agrega entrenamiento en vestuario y transferencias; } \\
\text { esto con el propósito de estructurar rutina, mantener nivel de independencia } \\
\text { funcional y promover sentimientos de utilidad. }\end{array}$ \\
\hline $\begin{array}{l}\text { Estimulación motora de las } \\
\text { extremidades superiores } \\
\text { EMEESS) }\end{array}$ & $\begin{array}{l}\text { Consiste en mantener o activar movimientos funcionales y la fuerza de EESS } \\
\text { por medio de actividades tales como: ejercicios con implementos, ergoterapias } \\
\text { y actividades bimanuales entre otros. }\end{array}$ \\
\hline Participación de la familia \\
$\begin{array}{l}\text { Se busca enriquecer la interacción entre la familia y el paciente, y la } \\
\text { colaboración de ésta en estrategias de estimulación cognitiva. Para ello se } \\
\text { realizan reuniones con la familia y se pone a su disposición material para } \\
\text { utilizar durante las visitas. }\end{array}$ \\
\hline
\end{tabular}

La recolección de datos y evaluaciones en las distintas etapas del estudio, fue encomendada a Terapeutas Ocupacionales y Kinesiólogos previamente capacitados en el screening realizado dentro de las primeras 24 horas, previo consentimiento informado (aprobado por el Comité de Ética del HCUCH), se recogieron datos acerca de la información demográfica, motivo de consulta y se aplicaron las pautas de evaluación: Test del informador o SS - IQCODE (TIN), que arroja información sobre el declive cognitivo y funcional del paciente, al comparar su situación actual con la misma hace 10 años (Del-Ser, 1997). Con respecto a la funcionalidad de los pacientes, se utilizó el Cuestionario de Actividad Funcional Pfeffer (Quiroga, 2004), que evalúa el funcionamiento en actividades de la vida diaria de mayor complejidad como: manejo de dinero, de medicación y uso de transporte, recogiendo información del nivel de independencia previo a la hospitalización. Además, se aplicó el FIM -Medida de Independencia Funcional- (del primer día de hospitalización), instrumento que mide la intensidad de asistencia dada por una tercera persona, en base a 18 actividades de la vida diaria que se puntúan en una escala desde el nivel 1 (dependencia completa) al nivel 7 (completa independencia), subdividiéndose en los 
ítems FIM- Motor con un puntaje máximo de 91 puntos y en un FIM-Cognitivo un puntaje máximo de 35 puntos (Young, 2009).

Una vez seleccionados los participantes que cumplieron con los criterios de inclusión, se determinó quienes serían del grupo control y grupo experimental de forma aleatoria, por un sujeto enmascarado.

Para valorar el objetivo principal del estudio, fueron evaluados ambos grupos respecto a la presencia o ausencia de delirium dos veces al día con el instrumento CAM (Confussion Assesment Method) por un máximo de 5 días desde el enrolamiento. El CAM determina la presencia o no de delirium en base a criterios establecidos (Inouye, 1990). Si la evaluación CAM resulta positiva a la presencia de delirium, se aplica la escala DRS (Delirium Rating Scale) para determinar su severidad, la que se cuantifica al sumar las puntuaciones (de 0 a 3 puntos) en 13 ítems, siendo 39 el puntaje máximo de severidad (Trzepacz, 1988).

Al 6to día y previo al alta se realizaron las siguientes evaluaciones: FIM para valorar cambios en la funcionalidad del paciente, MMSE (Mini-Mental State Examination), escala cognitivoconductual útil tanto para confirmar como para cuantificar el estado mental y fuerza de garra mediante el uso del Dinamómetro Jamar. En esta última, se realizaron tres mediciones en cada extremidad, con un intervalo de un minuto entre cada una para luego ser promediadas. Este aspecto, es considerado ya que ha demostrado ser un buen predictor de independencia, estado cognitivo y mortalidad en AM (Hamilton, 1992).

Dadas las características de la intervención, no se enmascaró al paciente ni al equipo que implementó la intervención. Esto si se realizó con el evaluador de los resultados, la randomización y el analista de la base de datos.

Se realizó el análisis y evaluación de los resultados a todas las variables del estudio mediante un análisis estadístico descriptivo, utilizando promedio y desviación estándar. Para la comparación del objetivo principal (incidencia de delirium), se utilizó el promedio entre el grupo de manejo estándar versus el de prevención reforzada, y para la evaluación funcional en los dominios motor y cognitivo del FIM, la fuerza de garra y la evaluación de estado cognitivo con MMSE, se empleó la prueba t- Student a dos colas. Para la comparación del subanálisis (presencia de delirium vs no presencia en grupo control) se utilizó la prueba U de Mann-Whitney. El nivel de significancia utilizado en el análisis fue $\mathrm{p}<0,05$. 


\section{RESULTADOS:}

De un total de 912 pacientes AM ingresados a UPC del HCUCH entre los meses de abril y octubre, 806 fueron excluidos ya que no cumplían con los criterios de inclusión. 70 pacientes aceptaron participar del estudio (35 grupo control y 35 grupo experimental). De los 35 pacientes del grupo control, uno de ellos declinó antes de comenzar la intervención, uno falleció durante la misma y dos fueron dados de alta sorpresivamente. Del grupo experimental de 35 pacientes, uno declina antes de comenzar la intervención y un paciente aún continúa con intervención de Terapia Ocupacional. (Figura $\mathrm{N}^{\mathrm{o}} 1$ )

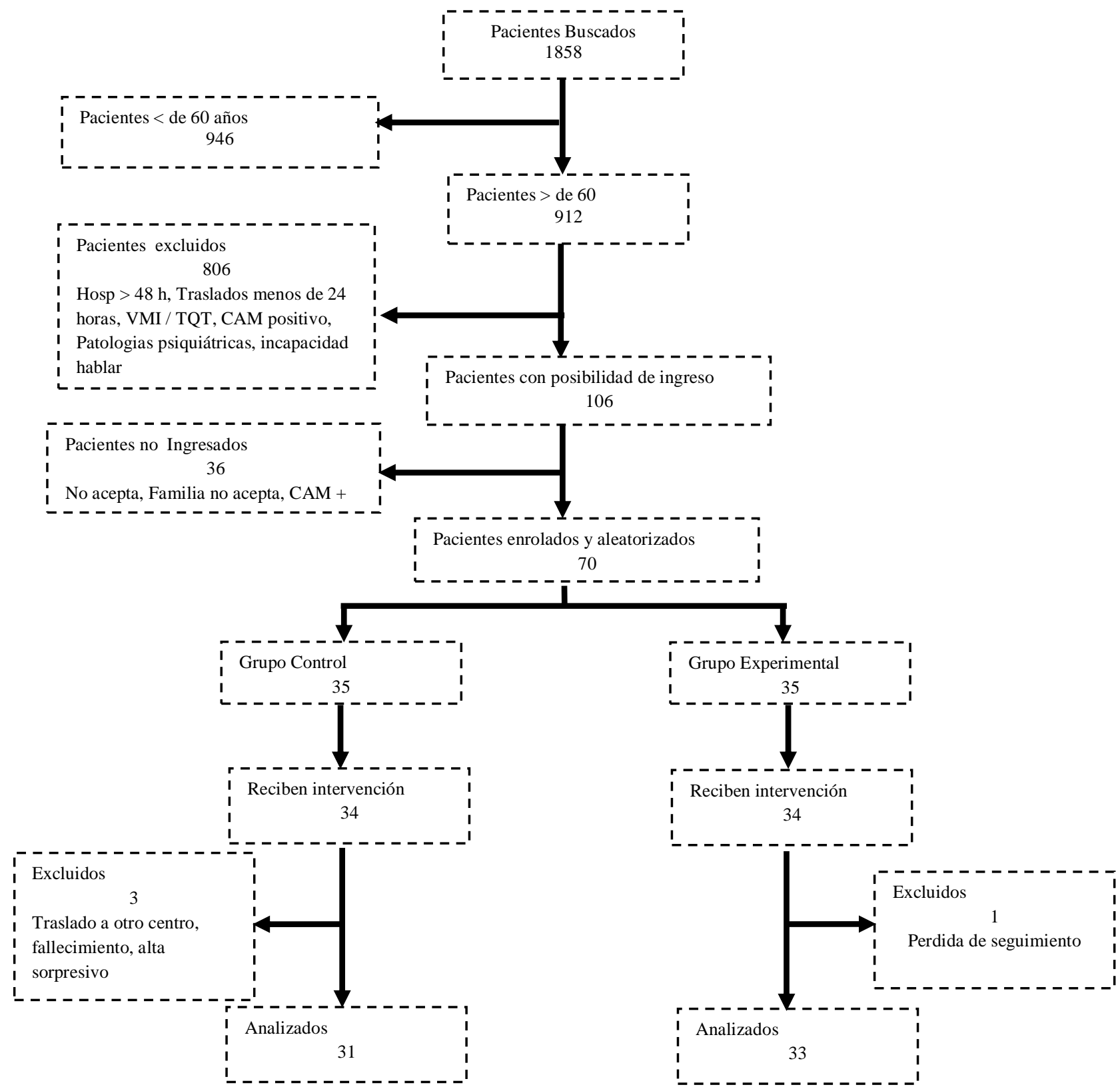

Figura 1: Diagrama de flujo de selección de la muestra. 
Ambos grupos presentaron similares condiciones de edad, sexo, estado civil y años de escolaridad, así también en el nivel de gravedad y disfunción orgánica, estado cognitivo e independencia funcional previa. Siendo los diagnósticos principales al ingreso: sepsis de foco urinario/ pulmonar y cáncer, con una distribución similar entre ambos grupos. (Tabla 2)

Tabla 2. Características basales de los pacientes de acuerdo al grupo de estudio.

\begin{tabular}{lcccc}
\hline Parámetros & $\begin{array}{c}\text { Total } \\
(\mathbf{N}=\mathbf{6 4})\end{array}$ & $\begin{array}{c}\text { Grupo Control } \\
(\mathbf{n = 3 1 )}\end{array}$ & $\begin{array}{c}\text { Grupo Experimental } \\
(\mathbf{n = 3 3 )}\end{array}$ & $\boldsymbol{P}^{\mathbf{1}}$ \\
\hline Edad \pm DE & $72,3 \pm 8,1$ & $73,1 \pm 7,9$ & $71,5 \pm 8,3$ & $\mathrm{~ns}$ \\
Sexo femenino (\%) & $28(43,7)$ & $15(48,4)$ & $13(39,4)$ & $\mathrm{ns}$ \\
Casados (\%) & $42(65,6)$ & $22(70,9)$ & $20(60,6)$ & $\mathrm{ns}$ \\
Años escolaridad \pm DE & $10,6 \pm 5,2$ & $10,9 \pm 5,9$ & $10,4 \pm 4,5$ & $\mathrm{~ns}$ \\
Estudios superiores (\%) & $18(28,1)$ & $10(32,2)$ & $8(24,2)$ & $\mathrm{ns}$ \\
Jubilados (\%) & $22(34,4)$ & $10(32,2)$ & $12(36,4)$ & $\mathrm{ns}$ \\
APACHE II \pm DE & $8,8 \pm 3,6$ & $9,1 \pm 3,7$ & $8,6 \pm 3,5$ & $\mathrm{~ns}$ \\
SOFA DE & $1,8 \pm 1,9$ & $2,1 \pm 2$ & $1,4 \pm 1,8$ & $\mathrm{~ns}$ \\
TIN DE & $80,7 \pm 11,4$ & $81,7 \pm 13,5$ & $79,9 \pm 8,9$ & $\mathrm{~ns}$ \\
PFEFFER \pm DE & $2,6 \pm 4,2$ & $3,2 \pm 5,2$ & $1,9 \pm 2,9$ & $\mathrm{~ns}$ \\
FIM motor inicial \pm DE & $20,5 \pm 6,2$ & $20,9 \pm 6,3$ & $20 \pm 6,2$ & $\mathrm{~ns}$ \\
FIM cognitivo inicial \pm DE & $52,6 \pm 22,3$ & $32,3 \pm 4,1$ & $33,0 \pm 3,0$ & $\mathrm{~ns}$ \\
Principales diagnósticos (\%) & & & & \\
Sepsis & $23(35,9)$ & $12(38,7)$ & $11(33.3)$ & $\mathrm{ns}$ \\
Cáncer & $16(2,5)$ & $10(32,3)$ & $6(18,1)$ & $\mathrm{ns}$ \\
Diabetes & $4(6,3)$ & $2(6,5)$ & $2(6)$ & $\mathrm{ns}$ \\
HTA & $3(4,7)$ & $1(3,2)$ & $2(6)$ & $\mathrm{ns}$ \\
EPOC & $3(4,7)$ & $1(3,2)$ & $2(6)$ & $\mathrm{ns}$ \\
Obstrucción intestinal & $3(4,7)$ & $0(0)$ & $3(9)$ & $\mathrm{ns}$ \\
Otros & $12(35,2)$ & $5(16,1)$ & $7(21,2)$ & $\mathrm{ns}$ \\
& & & & \\
\hline
\end{tabular}

Abreviaciones: APACHE, Acute Physiology and Chronic Helth Evaluation; SOFA, Secuential Organ Failure Assessment; TIN, Test del Informador; PFEFFER, Portable Fuctional Assessment Questionnaire; FIM, Functional Independence Measure; HTA, Hipertensión Arterial; EPOC, Enfermedad Pulmonar Obstructiva Crónica.

$\mathrm{P}^{1}$; comparaciones entre grupo control y grupo experimental.

En relación a los días de hospitalización, estos fueron significativamente mayores en pacientes del grupo control en comparación con el experimental (20,6 días versus 10,4 días. $\mathrm{P}=.009)$. (Gráfico 1) 


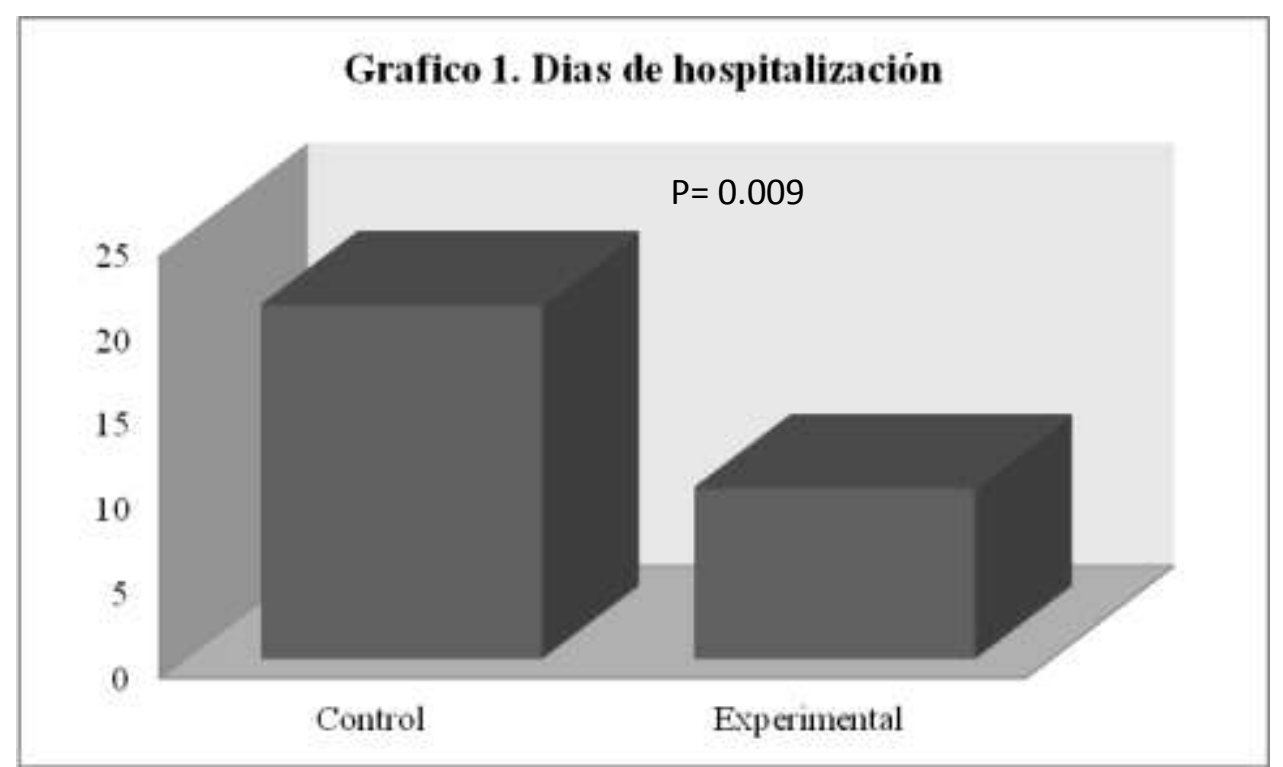

Gráfico 1. Días de hospitalización PnFE vs PnFR. Comparación de dias de hospitalizacion entre grupo control y grupo experimental. El análisis incluye a pacientes adultos mayores en UPC, 33 pacientes de GE y 31 pacientes en GC, presentando este último una mayor cantidad de dias de hospitalización.

\section{Eficacia de la intervención.}

Cinco personas del grupo control presentaron delirium durante la hospitalización en comparación a una del grupo experimental (diferencia estadísticamente significativa), presentando ambos grupos el mismo grado de severidad (Tabla 3, Gráfico 2).

\begin{tabular}{|c|c|c|c|c|c|c|}
\hline Parámetros & 1 & 2 & 3 & 4 & 5 & 6* \\
\hline Edad & 83 & 70 & 74 & 64 & 80 & 76 \\
\hline APACHE & 9 & 6 & 6 & 6 & 5 & 10 \\
\hline SOFA & 5 & 0 & 2 & 0 & 1 & 3 \\
\hline PFEFFER & 4 & 0 & 1 & 1 & 0 & 9 \\
\hline TIN & 77 & 77 & 87 & 78 & 83 & 95 \\
\hline Días hospitalización & $12^{1}$ & 58 & 39 & 68 & 17 & 40 \\
\hline FIM Motor Inicial & 15 & 15 & 15 & 14 & 21 & 13 \\
\hline FIM Cognitivo Inicial & 21 & 35 & 35 & 35 & 33 & 32 \\
\hline FIM Motor final & 22 & 13 & 17 & 19 & 31 & 18 \\
\hline FIM Cognitivo final & 33 & 29 & 31 & 29 & 30 & 27 \\
\hline MMSE & 27 & 22 & 27 & 22 & 25 & 24 \\
\hline Fuerza de garra & 4,6 & 13,5 & 13,6 & 17,6 & 6 & 9 \\
\hline № evaluaciones (+) CAM & 8 & 6 & 2 & 3 & 1 & 5 \\
\hline DRS & 10 & 11 & 11 & 14 & 12 & 12 \\
\hline
\end{tabular}

${ }^{1}$ Fallecido.

*Paciente grupo experimental. 


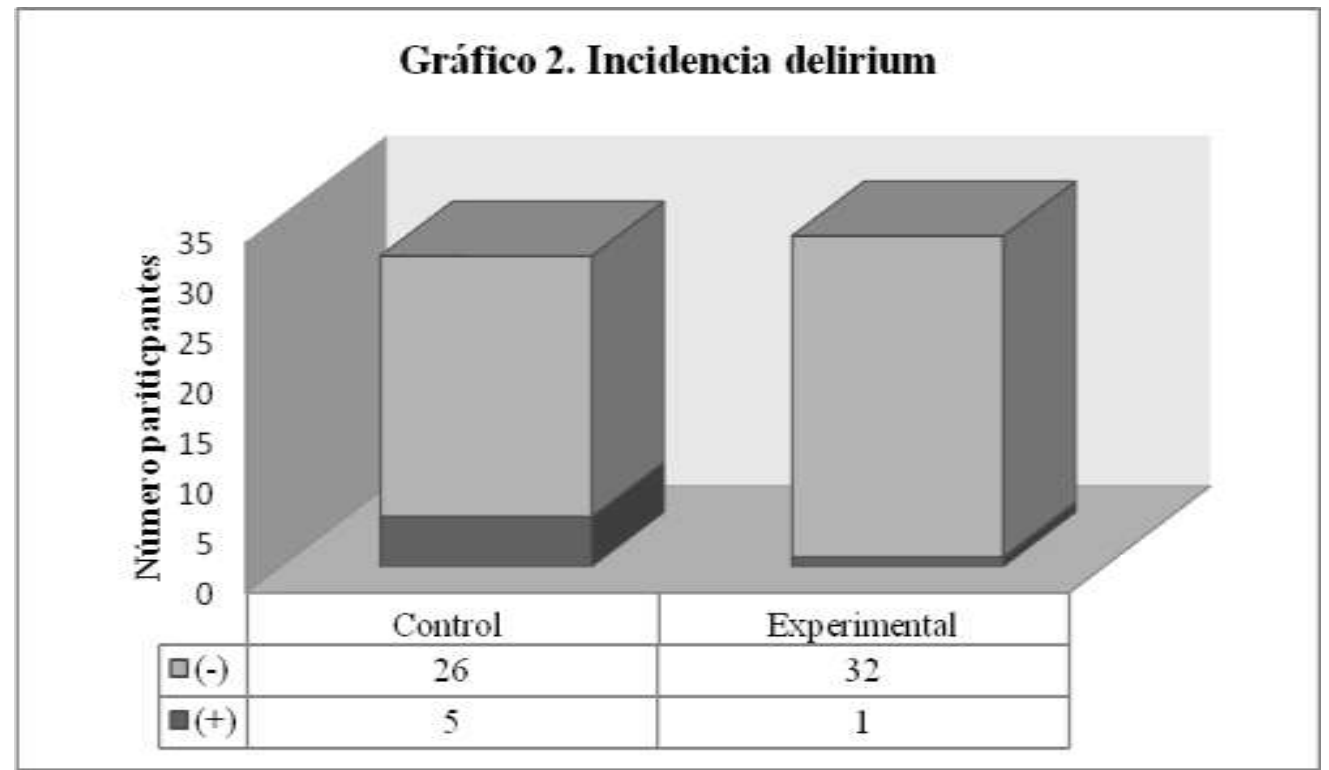

Gráfico 2. Comparación de la incidencia de delirium entre grupo control y grupo experimental. Se observan diferencias significativas entre ambos grupos, presentando el grupo control una mayor incidencia.

Al realizar un subanálisis entre los pacientes con y sin delirium del grupo control, se observó que los pacientes con delirium tuvieron más días de hospitalización y menor independencia funcional al alta en relación a los restantes del mismo grupo. (Tabla 4).

\section{Tabla 4. Grupo control delirium (+) vs delirium (-)}

\begin{tabular}{lccc|}
\hline Parámetros & $\begin{array}{c}\text { Delirium }(-) \\
(\mathbf{n = 2 6 )}\end{array}$ & $\begin{array}{c}\text { Delirium (+) } \\
(\mathbf{n}=\mathbf{5})\end{array}$ & $\boldsymbol{P}^{\mathbf{1}}$ \\
\hline Edad $\pm \mathrm{DE}$ & $72,8 \pm 8,2$ & $74,4 \pm 7,5$ & $\mathrm{Ns}$ \\
APACHE II $\pm \mathrm{DE}$ & $9,6 \pm 3,8$ & $6,4 \pm 1,5$ & $.03^{*}$ \\
SOFA \pm DE & $2,2 \pm 2$ & $1,8 \pm 1,9$ & $\mathrm{Ns}$ \\
PFEFFER \pm DE & $3,6 \pm 5,6$ & $1,2 \pm 1,6$ & $\mathrm{Ns}$ \\
Días hospitalización \pm DE & $17,3 \pm 17,4$ & $37,8 \pm 23,5$ & $.02^{*}$ \\
FIM Motor Inicial \pm DE & $21,8 \pm 6,3$ & $16 \pm 2,8$ & $.05^{*}$ \\
FIM Cognitivo Inicial $\pm \mathrm{DE}$ & $32,4 \pm 3,7$ & $31,8 \pm 6,1$ & $\mathrm{Ns}$ \\
FIM Motor final $\pm \mathrm{DE}$ & $48,7 \pm 20,5$ & $35,2 \pm 4$ & $\mathrm{Ns}$ \\
FIM Cognitivo final $\pm \mathrm{DE}$ & $32,5 \pm 18,7$ & $32 \pm 2,5$ & $\mathrm{Ns}$ \\
MMSE \pm DE & $25,4 \pm 4,1$ & $26,6 \pm 2,9$ & $\mathrm{Ns}$ \\
Fuerza de garra $\pm \mathrm{DE}$ & $20,4 \pm 11,2$ & $11,1 \pm 5,5$ & $\mathrm{Ns}$ \\
\hline
\end{tabular}

$\mathrm{P}^{\mathbf{1}}$; comparaciones entre pacientes con y sin delirium de grupo control; ns, no significativo. 
El estado cognitivo y la fuerza de garra no presentan importantes variaciones entre el grupo control y experimental (Tabla 5).

\begin{tabular}{|lccc|}
\hline Tabla 5. Resultados finales generales. & & \\
\hline \multicolumn{1}{l}{ Evaluaciones } & $\begin{array}{c}\text { Grupo Control } \\
(\mathbf{n}=\mathbf{3 1})\end{array}$ & $\begin{array}{c}\text { Grupo Experimental } \\
(\mathbf{n = 3 3 )}\end{array}$ & $\boldsymbol{P}^{\mathbf{1}}$ \\
\hline MMSE $\pm \mathrm{DE}$ & $25,6 \pm 3,9$ & $26,7 \pm 2,9$ & $\mathrm{Ns}$ \\
Fuerza de garra $\pm \mathrm{DE}$ & $18,8 \pm 11,0$ & $21,7 \pm 10,0$ & $\mathrm{Ns}$ \\
FIM motor final $\pm \mathrm{DE}$ & $46,5 \pm 20,5$ & $58,3 \pm 22,6$ & .03 \\
FIM cognitivo final $\pm \mathrm{DE}$ & $32,5 \pm 3,8$ & $32,9 \pm 5,9$ & $\mathrm{Ns}$ \\
Días de delirium & $2,2 \pm 1,3$ & 3 & $\mathrm{Ns}$ \\
DRS & 12 & 12 & $\mathrm{Ns}$ \\
Días hospitalización $\pm \mathrm{DE}$ & $20,6 \pm 19,6$ & $10,4 \pm 7,5$ & 0.09 \\
\hline
\end{tabular}

Tabla 5. Resultados Finales, muestra las evaluaciones finales, previas al alta de ambos grupos. Abreviaciones: MMSE, Mini-Mental State Examination; FIM, Functional Independence Measure; CAM, Confusion Assessment Method; DRS, Delirium Rating Scale. P1; comparaciones entre grupo control y grupo experimental.

La independencia funcional en AVDB al egreso no muestra diferencias en FIM Cognitivo, mientras que en el FIM Motor se observan mejores resultados en el grupo experimental, los cuales son significativos estadísticamente (p 0.03). Al comparar los resultados de FIM inicial y final, tanto en el grupo control como experimental existe un aumento de la independencia funcional, siendo mayor en este último. (Gráfico 3)

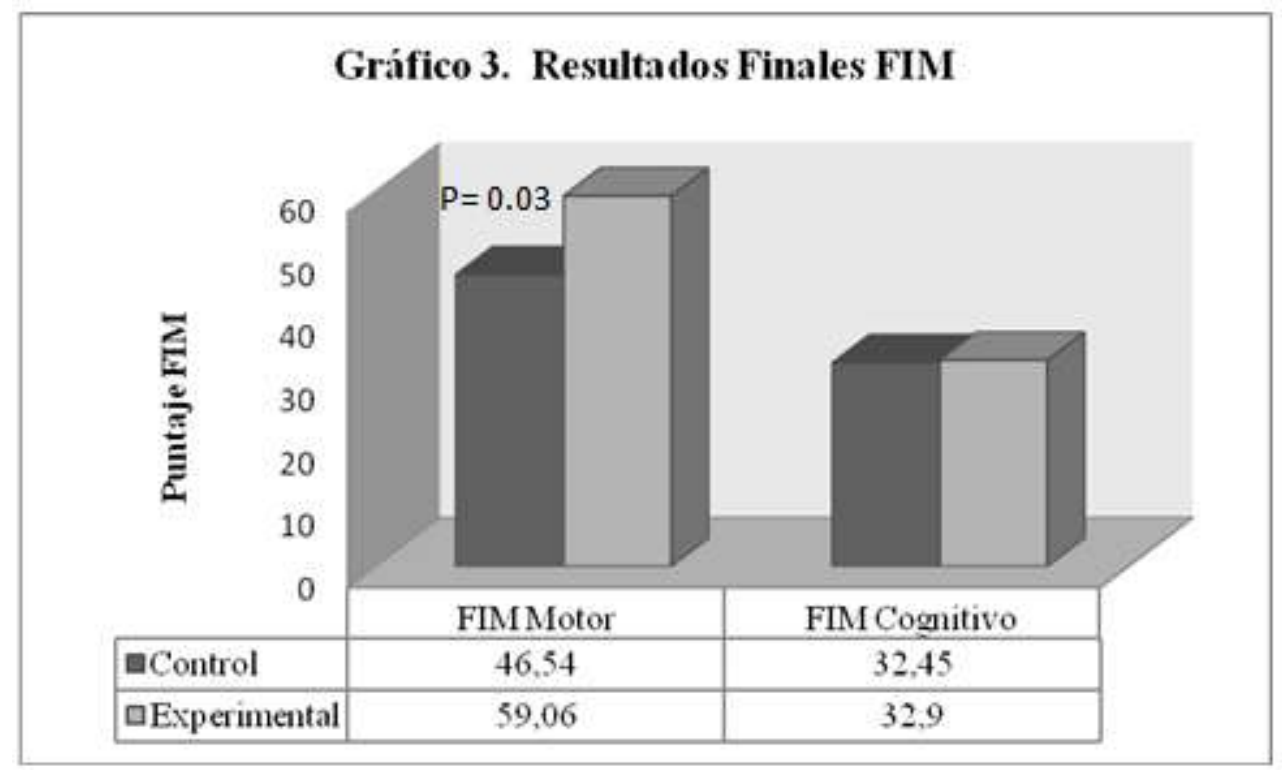

Gráfico 3. Resultados FIM final: Comparación de FIM realizado al final de la intervencion entre grupo control y grupo experimental. Se observan diferencias solo en FIM motor, obteniedo el grupo experimental un mayor puntaje que el grupo control. 
En la Tabla 6 se muestra la distribución del FIM según rango etario donde se observa que en el rango entre 60- 69 y 90-99 años existe similitud en ambos grupos, en cambio en el rango 70- 79 y 80-89 años, el resultado del FIM aumenta en el grupo experimental. En ambos grupos el nivel de funcionalidad disminuye en el último grupo etario (90-99).

\begin{tabular}{|lccc|}
\hline Tabla 6. FIM motor final según rango etario. & & \\
\hline & $\begin{array}{c}\text { FIM motor final } \\
\text { Grupo Control } \\
\text { (n=31) }\end{array}$ & $\begin{array}{c}\text { FIM motor final } \\
\text { Grupo Experimental } \\
(\mathbf{n}=\mathbf{3 3 )}\end{array}$ & $\boldsymbol{P}^{\mathbf{1}}$ \\
Rango etario & $54,1 \pm 21,6$ & $59,7 \pm 22,5$ & $\mathrm{~ns}$ \\
\hline $60-69 \pm$ SD & $47,7 \pm 20,5$ & $62,3 \pm 22,8$ & $\mathrm{~ns}$ \\
$70-79 \pm$ SD & $38,5 \pm 17,1$ & $51,2 \pm 22,5$ & $\mathrm{~ns}$ \\
$80-89 \pm$ SD & 25 & 25 & $\mathrm{~ns}$ \\
$90-99 \pm$ SD & & & \\
\hline
\end{tabular}

$\mathrm{P}^{1}$; comparaciones entre grupo control y grupo experimental.

\section{DISCUSIÓN:}

En el presente estudio, la intervención de Terapia Ocupacional precoz e intensiva, integrada a la práctica clínica diaria, redujo la presencia de delirium en AM hospitalizados por enfermedades agudas y su estadía hospitalaria, sin embargo, una vez manifestado el delirium no hay diferencia significativa en su severidad ni duración, observado en el único caso presentado hasta la fecha. (Tabla 5).

Los resultados para presencia de delirium, tanto en grupo control como en el experimental (16\% y $3 \%$ respectivamente), fueron significativamente menores a las cifras de incidencia para AM hospitalizados; referenciadas, estimadas entre un 14-56\% (Taekema, 2010). Esto se relaciona con que, ambos grupos recibieron prevención no farmacológica para el delirium, siendo la reforzada de mayor eficacia, constituyendo así una estrategia efectiva y de bajo costo, que impacta en la salud de los AM que se hospitalizan. Esto, debido a que logra prevenir las complicaciones asociadas a la presencia de delirium, así como también disminuir los días de estadía hospitalaria y el declive funcional asociado a ésta. Lo mismo ocurre con la fuerza de garra y el estado cognitivo (MMSE) al alta, ambos predictores del nivel de funcionalidad en AVDB, los que muestran resultados dentro de los rangos de normalidad para el grupo etario, aspecto no frecuente en AM hospitalizados (Ling, 2010; González, 2003).

El nivel de independencia funcional al alta, también fue evaluado. En concordancia con la propuesta de la OMS (1985), que postula la funcionalidad como concepto clave dentro de la definición de salud para el AM (Sanhueza, 2005), en la investigación, se observó una relación entre un buen nivel de funcionalidad previo y una menor incidencia de delirium, mientras que no se observó relación entre mayor edad y mayor incidencia de delirium. En este sentido, no se observa mayor variación en aspectos cognitivos en ambos grupos, relacionado con el criterio de exclusión de no poseer deterioro cognitivo previo.

En cuanto al resultado del FIM motor al alta, este aumentó en ambos grupos, logrando el grupo experimental un nivel significativamente mayor de funcionalidad que el control. Asimismo, se observa un descenso en el puntaje a medida que aumenta la edad en ambos grupos. 
Se observa que la presencia de delirium se asocia a un menor puntaje en el FIM motor inicial, en base a lo cual se considera que la dependencia en AVDB es un factor predictor para el desarrollo de delirium. Respecto a factores no modificables en la prevención del delirium, como la edad, se esperaría que los pacientes con delirium fueran los más añosos y aquellos más gravemente enfermos (Cole, 2004; Abbasi, 2009), sin embargo, en el presente estudio no se observó relación con factores como edad o resultados de SOFA y APACHE, ya que pacientes añosos con buen nivel de independencia previo, no tuvieron mayor riesgo de presentar delirium, siendo nuevamente un dato interesante de analizar, ya que el nivel de independencia pareciera ser un factor protector en la presencia de delirium.

Un aspecto que podría haber sido relevante de considerar en este estudio, es el nivel de satisfacción que presentan los usuarios o sus familiares frente a la intervención realizada, así como que áreas de la intervención resultan más significativas para ellos, con el fin de incorporar estas sugerencias en futuras intervenciones o manuales que pudieran surgir a partir de esta investigación.

A partir de los resultados obtenidos en la presente investigación, se debe destacar el aporte de TO en la prevención no farmacológica del delirium, como una profesión centrada en el cliente, que promueve la salud y bienestar a través de la ocupación. Esta se orienta a mejorar tanto la independencia como la calidad de vida de las personas, interviniendo en aspectos del sujeto, ambiente y la ocupación.

Durante toda la investigación, queda en evidencia la importancia de la intervención enfocada al sujeto con base en la ocupación. Sin embargo, también fueron de suma relevancia las acciones enfocadas al contexto. Una vez que el paciente ingresa al sistema hospitalario, se ve expuesto a una serie de intervenciones invasivas, a una modificación brusca de su entorno y rutina cotidiana, que aumentan su condición de fragilidad (Fried, 2004), pudiendo desencadenar eventos de salud no esperables como el delirium. En éste contexto la intervención de Terapia Ocupacional puede ayudar a disminuir esta condición de fragilidad, potenciando habilidades cognitivas, funcionales y sociales durante la hospitalización.

Por esto consideramos importante profundizar en el área hospitalaria de la Terapia Ocupacional y generar estrategias para validar y crear espacios de actuación, ya que con la visión integral que la caracteriza, puede realizar significativas contribuciones a la práctica clínica. Ante esta realidad se plantea una pregunta para futuras investigaciones ¿Cómo se posicionan los TO frente a esta nueva área de intervención?

\section{CONCLUSIÓN}

La intervención precoz e intensiva de Terapia Ocupacional en pacientes adultos mayores hospitalizados redujo la incidencia del delirium, los días de estadía hospitalaria y el gasto en días-cama. Además, la TO mejoró la independencia funcional al alta, influyendo a nivel global en la salud de los AM hospitalizados en UPC.

\section{Agradecimientos:}

Al Hospital Clínico de la Universidad de Chile y a todos los pacientes que en forma voluntaria participaron de la investigación, ya que sin ellos no hubiese sido posible la realización de esta. 


\section{REFERENCIAS BIBLIOGRÁFICAS}

- Abbasi M, Mohammadi E, Sheaykh A. Effect of a regular family visiting program as an affective, auditory, and tactile stimulation on the consciousness level of comatose patients with a head injury. Jpn J Nurs Sci. 2009 Jun; 6 (1): 6-21.

- American Psychiatric Association: "Diagnostic and statistical manual of mental disorder" 4th ed. Rev. Washington, DC. American Psychiatric Association; 1994; 129.

- Cole MG. Delirium in elderly patients. Am J Geriatr Psychiatry 2004; 12: 7-21.

- Del-Ser T, Morales JM, Barquero MS, Cantón R, Bermejo F. Application of a Spanish version of the "Informant Questionnaire on Cognitive Decline in the Elderly" in the clinical assessment of dementia. Alzheimer Dis Assoc Disord. 1997 Mar; 11(1): 3-8.

- Fong T, et al. Nat. Rev. Neurol. 5. 2009; 210-220. ; doi:10.1038/nrneurol.2009.24.

- Fried LP et al. Untangling the concepts of disability, frailty and comorbidity: Implications for improved targeting and care. J Gerontol Med Sci. 2004; 59: 255-263.

- González M, Martínez G, Calderón J, Villarroel L, Yuri F, Rojas C, Jeria A, Valdivia G, Marín P, Carrasco M. Impact of delirium on short-term mortality in elderly inpatients: a prospective cohort study. Psychosomatics. 2009 May-Jun; 50 (3):234-238.

- González M, de Pablo J, Valdés M, Matrai S, Peri JM, Fuentes E. Delirium: A predictor of mortality in the elderly. Eur. J. Psychiat 2005.. Vol. 19, N. ${ }^{\circ}$ 3, (165-171).

- González M, De Pablo J, Valdés M. Delirum: la confusión de los clínicos. Rev Méd Chile 2003; 131: 1051-1060.

- Guerini F, Frisoni GB, Morghen S, Speciale S, Bellelli G, Trabucchi M. Clinical Instability as a Predictor of Negative Outcomes Among Elderly Patients Admitted to a Rehabilitation Ward. J Am Med Dir Assoc. 2010 Jul; 11(6):443-448.

- Hagsten B, Svensson O, Gardulf A. Early individualized postoperative occupational therapy training in 100 patients improves ADL after hip fracture: a randomized trial. Acta Orthop Scand. 2004 Apr; 75 (2): 177-183.

- Hamilton GF, McDonald C, Chenier TC. Measurement of grip strength: validity and reliability of the sphygmomanometer and jamar grip dynamometer. J Orthop Sports Phys Ther. 1992; 16(5); 215-219.

- Inouye SK. Prevention of Delirium in Older Hospitalized Patients. Ann Med. 2000 May; 32 (4); 257-263.

- Inouye SK, Bogardus ST, Charpentier PA, Leo-Summers L, Acampora D, Holford TR, Cooney LM. A Multicomponent Intervention to prevent delirium in hospitalized Older Patients. N Engl J Med. 1999 Mar 4; 340 (9): 669-676.

- Inouye SK, Van Dyck CH, Alessi CA, Balkin S, Siegal AP, Horwitz RL. Clarifying Confusion: The Confusion Assessment Method. A new method for detection of delirium. Ann Intern Med 1990; 113: 941-948.

- Legg L, Drummond A, Langhorne P. et al. Occupational therapy for patients with problems in personal activities of daily living after stroke: systematic review of randomised trials. BMJ. 2007 Nov 3; 335 (7626): 922. Epub 2007 Sep 27.

- Leslie DL, Marcantonio ER, Zhang Y, Leo-Summers L, Inouye SK. One-year health care costs associated with delirium in the elderly population. Arch Intern Med. 2008 Jan 14; 168 (1): 27-32. 
- Ling CH, Taekema D, de Craen AJ, Gussekloo J, Westendorp RG, Maier AB. Handgrip strength and mortality in the oldest old population: the Leiden 85-plus study. CMAJ. 2010 Mar 23; 182 (5):429-435.

- Lipowski ZJ: Delirium in the elderly patient. N Engl J Med 1989; 320:578-582.

- Lipowski Z.J MD, FRCP (C): "Delirium (Acute Confusional States)" The Journal of the American Medical Association, 1987; 258:1789-1792.

- McDowell JA, Mion, LC, Lydon, TJ, Inouye SK. A non pharmacologic sleep protocol for hospitalized older patients. J. Am. Geriatr. Soc. 1998. 46, 700-705

- Miller M. Evaluation and Management of Delirium in Hospitalized Older Patients. Am Fam Physician. 2008; 78(11):1265-1270.

- Needham DM, Korupolu R, Zanni JM, Pradhan P, Colantuoni E, Palmer JB, Broker RG, Fan E. Early physical medicine and rehabilitation for patients with acute respiratory failure: a quality improvement project. Arch Phys Med Rehabil. 2010 Apr; 91(4):536542.

- Pessoa D, Eidi M, Bastos A, Curiati J, Farfel J, Jacob W. Delirium in hospitalized elderly patients and post-discharge mortality. Clinics 2010; 65 (3): 251.

- Quiroga P, Albala C, Klaasen G. Validación de un test de tamizaje para el diagnóstico de demencia asociada a edad, en Chile. Rev. méd. Chile. 2004, vol.132, n.4; 467-478.

- Rudolph J, Inouye S, Jones R, Yang F, Fong T, Levkoff S, Marcantonio. Delirium: An Independent Predictor of Functional Decline After Cardiac Surgery. JAGS 58:643-649, 2010.

- Sanhueza M, Castro M, Merino J. Adultos mayores funcionales: un nuevo concepto en salud. Cienc. enferm. 2005. Concepción, v. 11, n. 2, dic.

- Schweickert WD, Pohlman MC, Kress JP et al. Early physical and occupational therapy in mechanically ventilated, critically ill patients: a randomised controlled trial. Lancet. 2009 May 30;373(9678):1874-1882

- Taekema DG, Gussekloo J, Maier AB, Westendorp RG, de Craen AJ. Handgrip strength as a predictor of functional, psychological and social health. A prospective populationbased study among the oldest old. Age Ageing. 2010 May; 39(3):331-337.

- Trzepacz PT, Baker RW, Greenhouse J. A symptom rating scale for delirium. Psychiatry Res 1988; 23:89-97.

- Young Y, Fan MY, Hebel JR, Boult C. Concurrent validity of administering the functional independence measure (FIM) instrument by interview. Am J Phys Med Rehabil. 2009 Sep; 88(9):766-770.

\section{Fuentes Electrónicas}

- Gobierno de Chile. SENAMA. Las Personas Mayores en Chile: Situación, avances y desafíos del envejecimiento y la vejez. 2009. Disponible en: http://www.globalaging.org/elderrights/world/2009/agingchile.pdf

- Gobierno de Chile. Superintendencia de Salud. Impacto del Envejecimiento en el gasto en salud: Chile 2002 - 2020. Departamento de Estudios y Desarrollo Julio 2006. Disponible en: http://www.supersalud.gob.cl/documentacion/569/articles4021 recurso 1.pdf 